\title{
Dopamine receptor antagonist thioridazine inhibits tumor growth in a murine breast cancer model
}

\author{
TAO YIN ${ }^{1}$, SISI HE ${ }^{1}$, GUOBO SHEN ${ }^{1}$, TINGHONG YE ${ }^{1}$, FUCHUN GUO $^{1}$ and YONGSHENG WANG ${ }^{1,2}$ \\ ${ }^{1}$ State Key Laboratory of Biotherapy/Collaborative Innovation Center of Biotherapy, West China Hospital; \\ ${ }^{2}$ Department of Thoracic Oncology, Cancer Center, West China Hospital, Sichuan University, Chengdu, Sichuan 610041, P.R. China
}

Received October 24, 2013; Accepted April 24, 2014

DOI: $10.3892 / \mathrm{mmr} .2015 .3967$

\begin{abstract}
Neuropsychological factors have been shown to influence tumor progression and therapeutic response. The present study investigated the effect of the dopamine receptor antagonist thioridazine on murine breast cancer. The anti-tumor efficacy of thioridazine was assessed using a murine breast cancer model. Cell apoptosis and proliferation were analyzed in vitro using flow cytometry (FCM) and the MTT assay, respectively. Western blot analysis was performed to assess Akt, phosphorylated (p)-Akt, signal transducer and activator of transcription (STAT) 3, p-STAT3 and p-p65 in tumor cells following treatment with thioridazine. The Ki67 index and the number of terminal deoxynucleotidyl transferase dUTP nick end labeling (TUNEL)-positive apoptotic cells were assessed in the tumor sections. Thioridazine was found to reduce tumor growth, inhibit tumor cell proliferation and induce apoptosis in a dose- and time-dependent manner in vitro. Thioridazine was also found to markedly inhibit tumor proliferation and induce tumor cell apoptosis in vivo as shown by the lower Ki67 index and increase in TUNEL-positive cells. In addition, thioridazine was observed to inhibit the activation of the canonical nuclear factor $\kappa$-light-chain-enhancer of activated B cells pathway and exert anti-tumor effects by remodeling the tumor stroma, as well as inhibit angiogenesis in the tumor microenvironment. In conclusion, thioridazine was found to significantly inhibit breast tumor growth and the potential for thioridazine to be used in cancer therapy may be re-evaluated and investigated in clinical settings.
\end{abstract}

\section{Introduction}

Neuropsychological stimulation has been shown to affect tumor progression and therapeutic response. Neural stimulation is associated with increased tumor incidence and metastasis (1).

Correspondence to: Dr Yongsheng Wang, Department of Thoracic Oncology, Cancer Center, West China Hospital, Sichuan University, 37 Guo Xue Xiang, Chengdu, Sichuan 610041, P.R. China

E-mail:wangys75@gmail.com

Key words: thioridazine, breast cancer, angiogenesis $\beta$-adrenergic activation of the cyclic adenosine monophosphate-protein kinase A signaling pathway has been reported to enhance tumor angiogenesis in vivo and promote malignant cell growth (2). $\beta$-blockers have been shown to reduce recurrence rates and mortality in patients with breast, melanoma and prostate cancer (3-5). A recent report found that cholinergic parasympathetic signaling regulates prostate cancer invasion (6). Furthermore, transplantation of beta-endorphin neurons into the hypothalamus has been reported to increase the activity of natural killer (NK) cells and macrophages, reduce inflammation and epithelial to mesenchymal transition (EMT) in tumor tissues and suppress mammary tumor growth and progression (7). Neuropsychological factors also influence the effect of anticancer therapies. Nonmyelinating Schwann cells are components of a hematopoietic niche and maintain hematopoietic stem cell (HSC) hibernation (8). Sympathetic nerves in the marrow of mice promote the survival of constituents of the stem cell niche which initiate recovery, thus chemotherapy-induced nerve injury in the bone marrow has been found to impair hematopoietic regeneration (9). Adrenergic nerve protection strategies, for example the administration of 4-methylcatechol or glial-derived neurotrophic factor, have been reported to promote hematopoietic recovery (9).

Dopamine receptors (DRs) are involved in several physiological and pathological processes, including age, emotion, opiate addiction and vascular activity. Five DRs have been identified and divided into two families: The D1 (including D1R and D5R) and D2 (including D2R, D3R and D4R) receptor families (10). DRs expressed on lymphocytes are involved in pathogenesis. D3R expressed on $\mathrm{CD}^{+} \mathrm{T}$ cells has also been reported to have an important role in the pathogenesis of 1-methyl-4-phenyl-1,2,3,6-tetrahydropyridine-induced Parkinson's disease (11). D3R-deficient mice were observed to be protected against the loss of dopaminergic neurons and microglial activation during MPTP-induced Parkinson's disease; however, upon transfer of wild-type $\mathrm{CD}^{+} \mathrm{T}$ cells, mice became susceptible to MPTP-induced neuronal degeneration and activation of microglia (11). The role of dopamine, not only in mediating interactions in the nervous system, but as an immunomodulator in the pathogenesis of disease, has been the focus of much research (12). Circulating dopamine levels have been reported to be higher in patients with lung cancer compared with healthy donors and dopamine has been 
found to effectively inhibit proliferation and cytotoxicity in T lymphocytes through D1 DRs (13).

Sachlos et al (14) identified that DRs are expressed in lymphoid stem cells and CD44 ${ }^{+}$CD24\%/low breast cancer stem cells (CSCs). However, DRs have not been observed in primitive HSCs or progenitor populations. Differential DR expression enables potential DR drug targeting for cancer (14). Thioridazine is a well established anti-psychotic and -anxiety agent which acts through DR1-5 (15). The present study aimed to investigate the anti-tumor effect of thioridazine in a murine breast cancer model.

\section{Materials and methods}

Cell culture. The 4T1 cancer cell line (American Type Culture Collection, Manassas, VA, USA) was maintained in RPMI-1640 (Gibco-BRL, Carlsbad, CA, USA) supplemented with $10 \%$ fetal bovine serum at $37^{\circ} \mathrm{C}$ in a humidified atmosphere containing $5 \% \mathrm{CO}_{2}$.

Cell cycle and apoptosis analysis. The 4T1 cell line was treated with 5, 10 and $20 \mu \mathrm{M}$ thioridazine. To assess apoptosis, cells were collected and stained with fluorescein isothiocyanate (FITC)-labeled Annexin V and propidium iodide, according to the manufacturer's instructions (Roche, Mannheim, Germany). Apoptotic cells were analyzed using flow cytometry (FCM) using CellQuest ${ }^{\mathrm{TM}}$ software (BD Biosciences, San Jose, CA, USA). Thioridazine was purchased from Sigma-Aldrich (St. Louis, MO, USA).

MTT assay. MTT assay was performed in 96-well plates to determine cell growth inhibition. A total of 3,000 cells/well were seeded in 96-well plates and treated with $20 \mu \mathrm{M}$ thioridazine the following day. After 24-72 h drug incubation, MTT was added to each well and incubated for $4 \mathrm{~h}$ at $37^{\circ} \mathrm{C}$. The supernatant was then removed and formazan precipitates in the cells were dissolved using $150 \mu$ l dimethyl sulfoxide. Absorbance was read at $570 \mathrm{~nm}$ and a growth curve was plotted.

Western blot analysis. Subsequent to treatment with $20 \mu \mathrm{M}$ thioridazine for $24 \mathrm{~h}, 4 \mathrm{~T} 1$ tumor cells were harvested and lysed using radioimmunoprecipitation assay buffer containing protease inhibitors. Equal quantities of protein were loaded and resolved using SDS-PAGE and transferred onto polyvinylidene difluoride membranes. Membranes were then incubated with primary antibodies against STAT3, phosphorylated (p)-STAT3, Akt, p-Akt and p-p65 (all rabbit mAb; cat. nos. 4904, 9145, 4685, 4051 and 3033 respectively; Cell Signaling Technology Inc., Danvers, MA USA), followed by incubation with horseradish peroxidase-conjugated secondary antibodies (Goat pAb; cat. no. SP-9001; ZSGB-BIO ORIGENE, Beijing, China). Immunoreactive bands were visualized using an enhanced chemiluminescence detection system.

Tumor challenge and treatment. In order to determine the anti-tumor activity of thioridazine, flank syngeneic breast tumors were established in six- to eight-week-old female BALB/c mice through subcutaneous injection with $1 \times 10^{6} 4 \mathrm{~T} 1$ cells. When the tumors reached $\sim 100 \mathrm{~mm}^{3}$ on day six following inoculation, the mice with established tumors were stratified by tumor volume and randomly divided into two groups, which were treated with vehicle and thioridazine $(32 \mathrm{mg} / \mathrm{kg}$ body weight), respectively. Thioridazine was intraperitoneally administered daily. Tumor diameters were measured using a caliper and tumor volumes were calculated by the formula $0.52 \mathrm{x} \mathrm{a} \mathrm{xb}^{2}$, where $\mathrm{a}$ is the larger diameter and $\mathrm{b}$ is the smaller diameter.

Immunohistochemistry. Tumors were harvested and paraffin-embedded, then tumor sections were stained with hematoxylin and eosin. For the analysis of cell proliferation, tumor sections were incubated with anti-Ki67 antibodies (cat. no. AB9260; Millipore Corporation, Billerica, MA, USA) at $4^{\circ} \mathrm{C}$ overnight, followed by incubation with biotin-conjugated secondary antibodies and streptavidin-horseradish peroxidase complexes. For the analysis of apoptosis, the TUNEL assay (Promega Corporation, Madison, WI, USA) was performed on the tumor sections, according to the manufacturer's instructions. Frozen tumor sections were incubated with CD31 antibodies (cat. no. ab7388; Abcam PLC, Cambridge, UK) and phycoerythrin-conjugated secondary antibodies (cat. no. ab7010; Abcam PLC) for microvessel analysis.

Statistical analysis. Data are presented as the mean \pm standard deviation. Statistical comparisons were performed using analysis of variance or the student's t-test. SPSS V13.0 software (SPSS Inc., Chicago, IL, USA) was used for statistical analysis. $\mathrm{P}<0.05$ was considered to indicate a statistically significant difference.

\section{Results}

Dopamine receptor antagonist thioridazine reduces breast tumor growth. To determine the anti-tumor efficacy of thioridazine in vivo, flank syngeneic $4 \mathrm{~T} 1$ breast tumors were established in BALB/c mice. The mice with then treated with vehicle or thioridazine. The control tumors were observed to grow rapidly, with a volume of $1232.8 \pm 282.8 \mathrm{~mm}^{3}$ at day 25 post-inoculation. However, following treatment with thioridazine, the tumor volume was found to be $549.3 \pm 205.2 \mathrm{~mm}^{3}$ (Fig. 1A and B). Thus, thioridazine reduced tumor volume by $55 \%$ (Fig. 1C), suggesting that the dopamine receptor antagonist thioridazine effectively reduces breast tumor growth.

Thioridazine induces apoptosis and inhibits tumor cell growth in vitro. To investigate the cytotoxic and cytostatic effects of thioridazine on breast cancer cells, murine 4T1 cells were treated with various concentrations of thioridazine for $24 \mathrm{~h}$. Cell apoptosis was then analyzed using FCM. Thioridazine was observed to markedly induce 4T1 cell apoptosis in a dose-dependent manner (Fig. 2A and B). Furthermore, an MTT assay revealed that thioridazine significantly inhibited tumor growth (Fig. 2C). These findings suggested that thioridazine effectively reduced tumor cell growth and induced tumor apoptosis.

Thioridazine induces apoptosis and inhibits tumor cell growth in vivo. In order to investigate whether thioridazine inhibits tumor cell proliferation in vivo, histological assessment was 
A

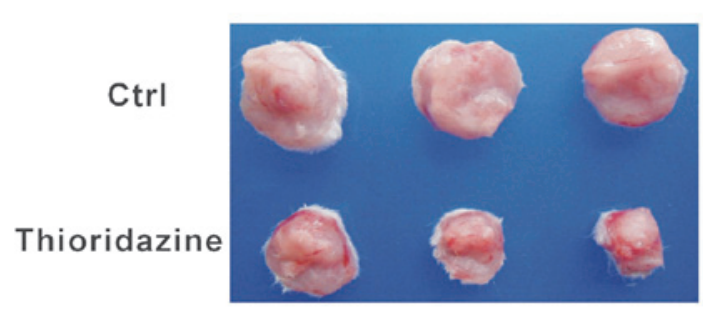

C

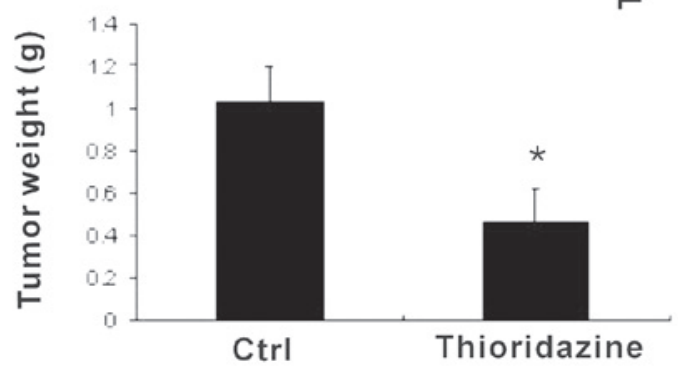

B

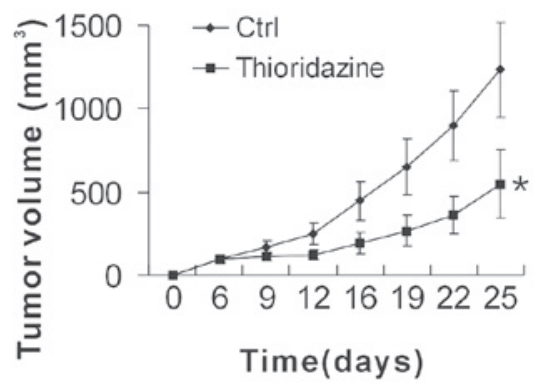

Figure 1. Dopamine receptor antagonist thioridazine reduces breast tumor growth. (A) Tumors from each group at day 25 post-inoculation. (B) Tumors were measured regularly and the relative tumor volumes were calculated. (C) Tumor weight was measured at day 25 post-inoculation and thioridazine significantly reduced tumor volume and weight. ${ }^{*} \mathrm{P}<0.05$ vs. ctrl. Ctrl, control.

A

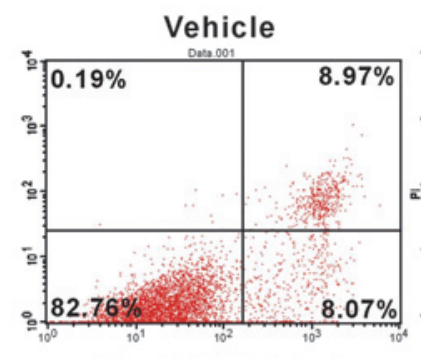

$10 \mu \mathrm{M}$ Thioridazine

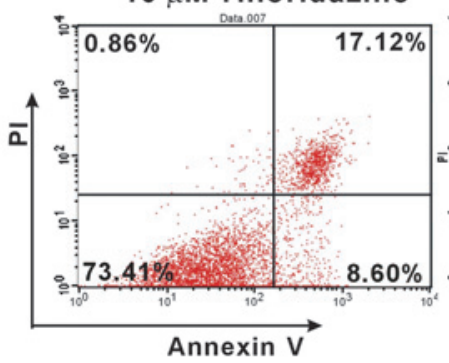

$5 \mu \mathrm{M}$ Thioridazine

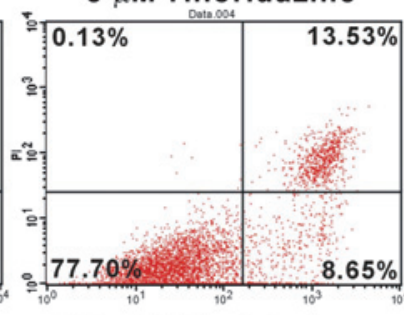

$20 \mu \mathrm{M}$ Thioridazine

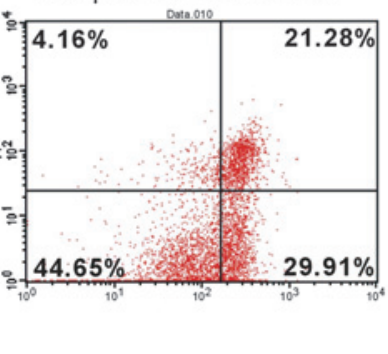

B

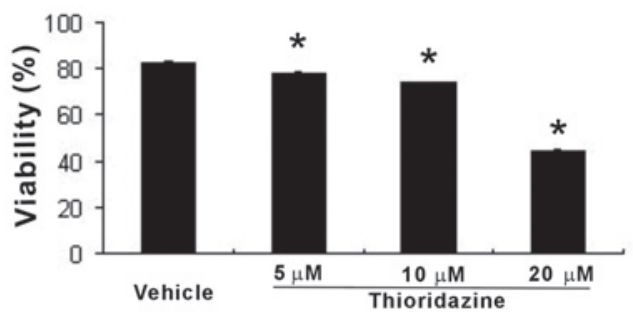

C

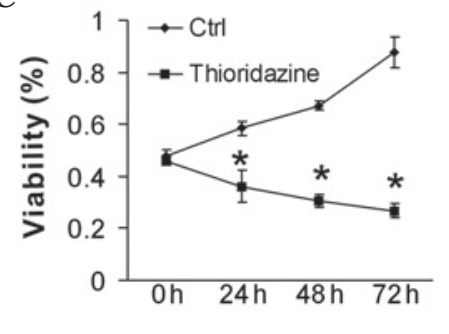

Figure 2. Thioridazine induces tumor cell apoptosis and inhibits tumor cell proliferation. (A and B) $4 \mathrm{~T} 1$ tumor cells were treated for 24 h with 5 , 10 and $20 \mu \mathrm{M}$ thioridazine. Tumor cell apoptosis was assessed using flow cytometry. Thioridazine induced cell apoptosis in a dose-dependent manner. (C) $4 \mathrm{~T} 1$ tumor cells were treated with $20 \mu \mathrm{M}$ thioridazine for 24,48 and $72 \mathrm{~h}$. Tumor cell viability was determined using the MTT assay. Thioridazine inhibited tumor cell proliferation in a time-dependent manner. ${ }^{*} \mathrm{P}<0.01$ vs. ctrl. Ctrl, control.

performed using tumor samples from 4T1 tumors. The 4T1 tumors from the mice in the control group showed poor differentiation and limited necrosis (Fig. 3, upper panel). By contrast, treatment with thioridazine was found to induce marked hemorrhagic necrosis (Fig. 3, upper panel). The TUNEL assay revealed that apoptosis was higher in the thioridazine treatment group compared with that in the control group (Fig. 3, middle panel). Furthermore, immunohistochemistry showed a decrease in cell proliferation in the thioridazine therapy group, as indicated by a decrease in the cell cycle marker Ki67, with the tumors from the control mice exhibiting higher proliferation (Ki-67+) indexes (Fig. 3, lower panel).
Thioridazine-induced suppression of the nuclear factor $\kappa$-light-chain-enhancer of activated B cells $(N F \kappa B)$ pathway in breast cancer cells. In order to determine the cell signaling pathways mediating the thioridazine-induced tumor inhibition in the 4T1 breast cancer cells, the levels of Akt, STAT3 and NFKB p65 were analyzed. As shown in Fig. 4, thioridazine was not observed to reduce the activity of Akt or STAT3 in 4T1 cells. However, thioridazine was found to significantly inhibit the phosphorylation of NFkB p65 (Fig. 4). These findings suggested that thioridazine inhibited cell proliferation and induced cell apoptosis through inhibiting the $N F \kappa B$ pathway. 


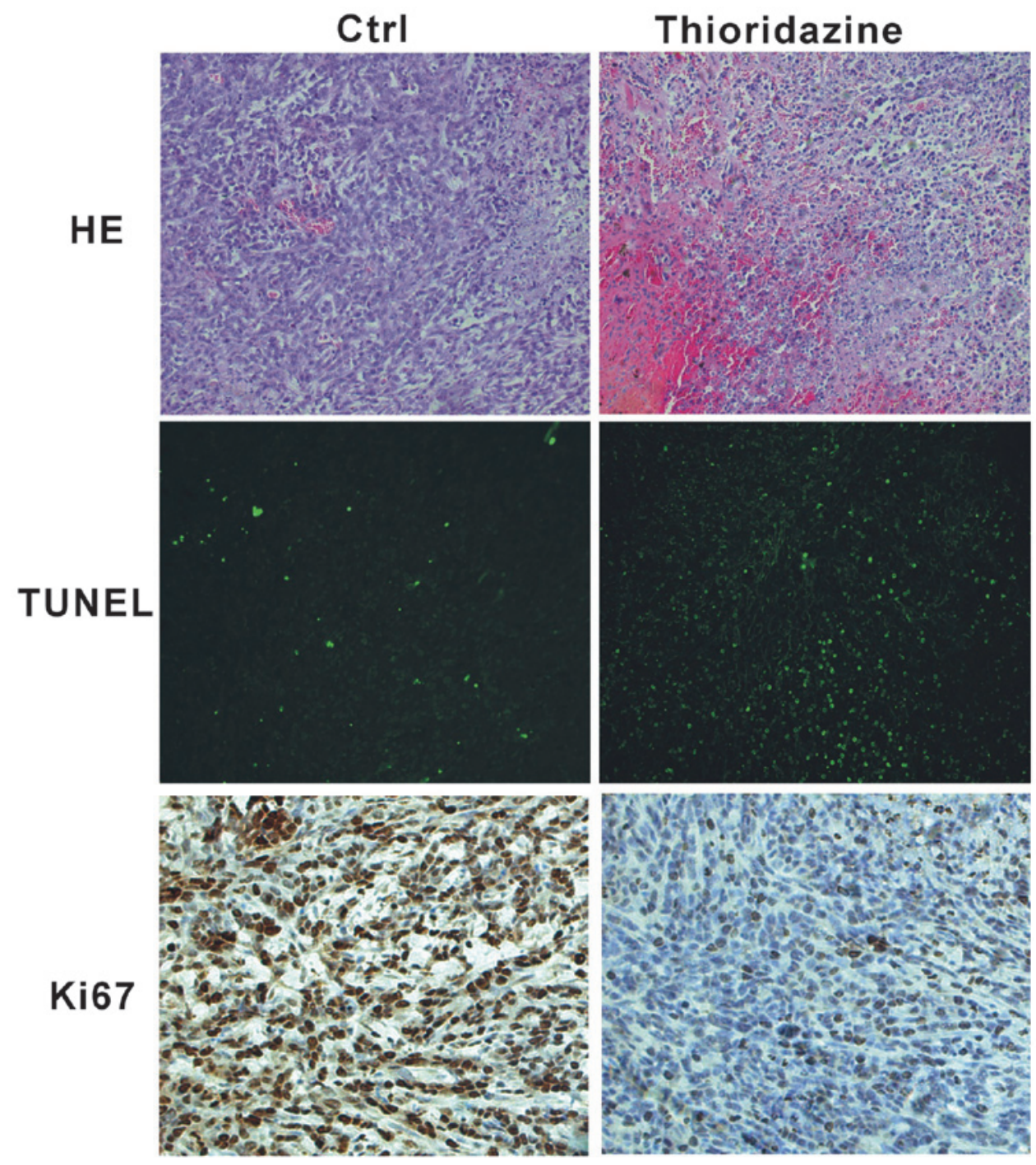

Figure 3. Thioridazine induces tumor necrosis and apoptosis and inhibits tumor cell proliferation. The upper panel shows HE-stained tumor sections, x200 magnification. Hemorrhagic necrosis was increased following treatment with thioridazine (right) compared with the control (left). The middle panel shows apoptosis detected using TUNEL staining. A higher frequency of apoptotic tumor cells was found in the tumors from the thioridazine-treated animals (right) compared with the controls (left). The lower panel shows tumor sections immunohistochemically stained for Ki67. A greater number of mitotic tumor cells was observed in the tumors from the control mice (left) compared with the tumors from the thioridazine-treated animals (right). HE, hematoxylin and eosin; TUNEL, terminal deoxynucleotidyl transferase dUTP nick end labeling; ctrl, control.

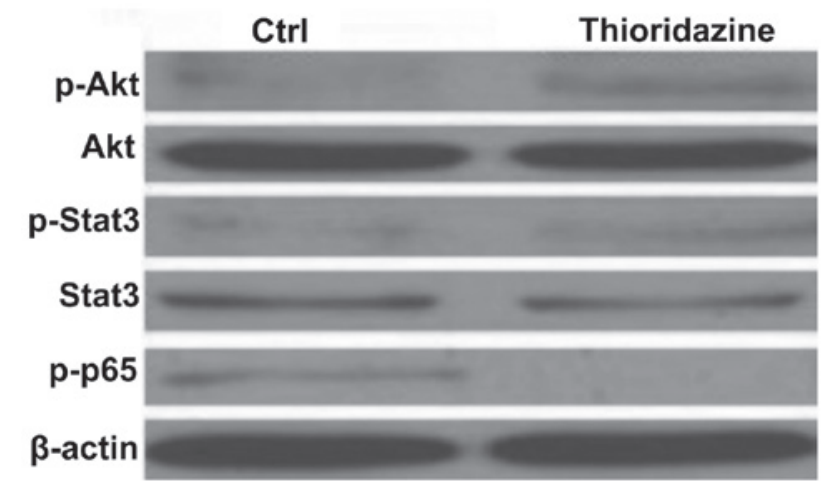

Figure 4. Thioridazine inhibits the NFkB pathway. 4T1 tumor cells were treated for $24 \mathrm{~h}$ with $20 \mu \mathrm{M}$ thioridazine. Western blot analysis was performed using 4T1 tumor cell lysates and anti-p-Akt, -Akt, -p-STAT3, -STAT3 and p-NFKB p65 antibodies. $\beta$-actin was used as a loading control. Thioridazine did not affect the phosphorylation of Akt and STAT3, while it markedly inhibited phosphorylation of NFkB p65. STAT, signal transducer and activator of transcription; $\mathrm{NF \kappa B}$, nuclear factor $\kappa$-light-chain-enhancer of activated B cells; p-, phosphorylated; ctrl, control.
Thioridazine inhibits tumor angiogenesis. In order to assess whether tumor blood vessel formation was inhibited by thioridazine treatment, CD $31^{+}$microvessels were analyzed using immunohistochemistry. Thioridazine was observed to markedly reduce vascularization compared with the control (Fig. 5A). Furthermore, the vessels in the thioridazine-treated tumors were found to be smaller in diameter than those in the control. Quantitative analysis of microvessel density revealed that thioridazine treatment had a significant inhibitory effect on tumor neovascularization (Fig. 5B). These findings suggested that thioridazine treatment inhibited tumor angiogenesis.

\section{Discussion}

The present study investigated the anti-tumor efficacy of the DR inhibitor thioridazine in a mouse breast cancer model. Thioridazine was observed to significantly reduce tumor 
A

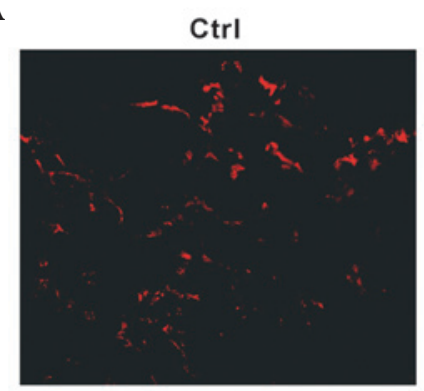

Thioridazine

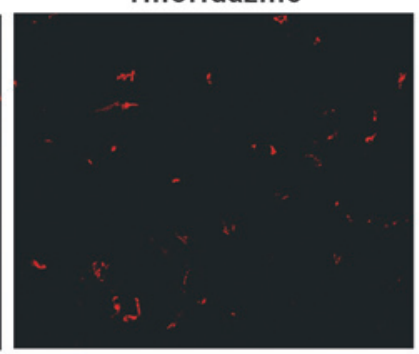

B

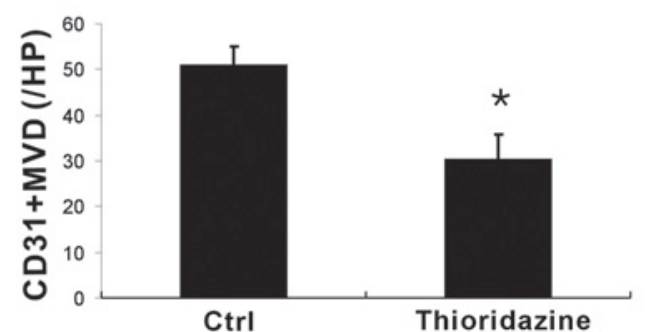

Figure 5. Thioridazine inhibits angiogenesis and reduces tumor vasculature. (A) Immunofluorecence staining with CD31 showed that more tumor vessels were present in the ctrl compared with the thioridazine-treated tumors. The vessels in thioridazine-treated tumors were smaller in diameter than those in the control tumors. (B) Quantification of CD31+ tumor vessels revealed that thioridazine significantly reduced tumor MVD. *P<0.05 vs. ctrl. Ctrl, control; MVD, microvessel density.

growth, inhibit proliferation and angiogenesis, as well as induce apoptosis. Furthermore, these thioridazine-induced effects were associated with inhibition of the canonical NFkB pathway.

Thioridazine has been extensively used to treat mental disorders. In the case of cancer, thioridazine has been used to treat tumor-associated depression (16) and sweating (17). In the present study, thioridazine was found to effectively suppress breast tumor growth in a preclinical model. Thioridazine has previously been identified to be an inhibitor of the phosphatidylinositol-3-kinase (PI3K)/Akt pathway in ovarian cancer cells (18). The PI3K pathway has an important role in tumorigenesis and therapeutic response (19). Almost every aspect of cell regulation, including apoptosis, proliferation, differentiation, oncogenic transformation, tumorigenesis and angiogenesis have been associated with PI3K activity (20). Thus, the present study investigated the effect of thioridazine on the inhibition of the PI3K/Akt pathway in breast cancer cells; however, thioridazine was not observed to affect Akt phosphorylation. Furthermore, thioridazine was not found to activate STAT3, another transcription factor associated with tumor proliferation and angiogenesis $(21,22)$. The $\mathrm{NF \kappa B}$ pathway has been identified to have a central role in apoptosis, proliferation and differentiation (23). The present study assessed the phosphorylation of the NFאB p65 subunit following treatment with thioridazine and found that thioridazine markedly reduced p65 phosphorylation. There are numerous NF- $\mathrm{KB}$-dependent targets which are involved in different aspects of tumorigenesis, including c-Myc, cyclinD1, cyclinE and cyclin-dependent kinase 2 which are involved in tumor cell proliferation, $\mathrm{X}$-linked inhibitor of apoptosis protein and survivin, which are involved in cell survival, matrix metalloproteinase 2/9 and intercellular adhesion molecule 1 , which are involved in metastasis, interleukin (IL)-1, inducible nitric oxide synthase and cyclooxygenase 2 , which are involved in inflammation, vimentin and twist, which are involved in EMT and vascular endothelial growth factor and IL-8, which are involved in angiogenesis (23). Several $\mathrm{NF \kappa B}$ pathway inhibitors have been shown to be effective for cancer therapy (24).

Angiogenesis is essential for tumor growth and metastasis and targeting angiogenesis is a promising strategy for cancer therapy (25). The present study showed that thioridazine is an angiostatic agent which was found to effectively inhibit angiogenesis in a murine tumor model. There have been several studies on the anti-angiogenic effect of thioridazine, which have reported findings consistent with the results of the present study. In vitro, thioridazine has been observed to inhibit vascular endothelial growth factor (VEGF)-induced proliferation, invasion and endothelial cell tube formation of (26). In the present study, thioridazine was found to reduce microvessel density in vivo. Focal adhesion kinase (FAK) is upregulated in various types of cancer and has been reported to regulate tumor angiogenesis, which has been associated with endothelial cell survival, proliferation and migration (27). Thioridazine has been found to exert its anti-angiogenic effects through the inhibition of integrin $\alpha \mathrm{V}$-mediated VEGF expression in tumor cells and the inhibition of endothelial cell migration by suppressing the phosphorylation of Src/FAK (26). Furthermore, the angiostatic properties of thioridazine may be partially mediated through the suppression of CSCs. DRs are expressed on the CSCs (14), which secrete VEGF and have high angiogenic activity (28).

In conclusion, the present study provided evidence that the DR antagonist thioridazine is an effective drug against breast cancer. The potential for this drug to be used in cancer therapy requires further investigation in clinical settings.

\section{Acknowledgements}

This study was supported by a grant from the National Natural Science Foundation of China (no. 81272523) and the National Major Project (no. 2011ZX09302-001-01).

\section{References}

1. Schuller HM, Al-Wadei H, Ullah MF and Plummer HK III: Regulation of pancreatic cancer by neuropsychological stress responses: a novel target for intervention. Carcinogenesis 33: 191-196, 2012.

2. Thaker PH, Han LY, Kamat AA, et al: Chronic stress promotes tumor growth and angiogenesis in a mouse model of ovarian carcinoma. Nat Med 12: 939-944, 2006.

3. Melhem-Bertrandt A, Chavez-MacGregor M, Lei X, et al: Beta-blocker use is associated with improved relapse-free survival in patients with triple-negative breast cancer. J Clin Oncol 29: 2645-2652, 2011

4. Lemeshow S, Sørensen HT, Phillips G, et al: $\beta$-blockers and survival among Danish patients with malignant melanoma: a population-based cohort study. Cancer Epidemiol Biomarkers Prev 20: 2273-2279, 2011. 
5. Grytli HH, Fagerland MW, Fosså SD, et al: Use of $\beta$-blockers is associated with prostate cancer-specific survival in prostate cancer patients on androgen deprivation therapy. Prostate 73: 250-260, 2013

6. Magnon C, Hall SJ, Lin J, et al: Autonomic nerve development contributes to prostate cancer progression. Science 341: 1236361, 2013.

7. Sarkar DK, Zhang C, Murugan S, et al: Transplantation of $\beta$-endorphin neurons into the hypothalamus promotes immune function and restricts the growth and metastasis of mammary carcinoma. Cancer Res 71: 6282-6291, 2011.

8. Yamazaki S, Ema H, Karlsson G, et al: Nonmyelinating Schwann cells maintain hematopoietic stem cell hibernation in the bone marrow niche. Cell 147: 1146-1158, 2011.

9. Lucas D, Scheiermann C, Chow A, et al: Chemotherapy-induced bone marrow nerve injury impairs hematopoietic regeneration. Nat Med 19: 695-703, 2013

10. Sibley DR, Monsma FJ Jr: Molecular biology of dopamine receptors. Trends Pharmacol Sci 13: 61-69, 1992.

11. González H, Contreras F, Prado C, et al: Dopamine receptor D3 expressed on $\mathrm{CD}^{+} \mathrm{T}$ cells favors neurodegeneration of dopaminergic neurons during Parkinson's Disease. J Immunol 190: 5048-5056, 2013.

12. Pacheco R, Prado CE, Barrientos MJ and Bernales S: Role of dopamine in the physiology of T-cells and dendritic cells. J Neuroimmunol 216: 8-19, 2009.

13. Saha B, Mondal AC, Basu S and Dasgupta PS: Circulating dopamine level, in lung carcinoma patients, inhibits proliferation and cytotoxicity of CD4+ and CD8+ T cells by D1 dopamine receptors: an in vitro analysis. Int Immunopharmacol 1: 1363-1374, 2001.

14. Sachlos E, Risueño RM, Laronde $S$, et al: Identification of drugs including a dopamine receptor antagonist that selectively target cancer stem cells. Cell 149: 1284-1297, 2012.

15. Seeman P and Lee T: Antipsychotic drugs: direct correlation between clinical potency and presynaptic action on dopamine neurons. Science 188: 1217-1219, 1975.
16. Ly KL, Chidgey J, Addington-Hall J and Hotopf M: Depression in palliative care: a systematic review. Part 2. Treatment. Palliat Med 16: 279-284, 2002.

17. Zhukovsky DS: Fever and sweats in the patient with advanced cancer. Hematol Oncol Clin North Am 16: 579-588, 2002.

18. Rho SB, Kim BR and Kang S: A gene signature-based approach identifies thioridazine as an inhibitor of phosphatidylinositol-3'-kinase (PI3K)/AKT pathway in ovarian cancer cells. Gynecol Oncol 120: 121-127, 2011.

19. Engelman JA: Targeting PI3K signalling in cancer: opportunities, challenges and limitations. Nat Rev Cancer 9: 550-562, 2009.

20. Osaki M. Oshimura M and Ito H: PI3K-Akt pathway: its functions and alterations in human cancer. Apoptosis 9: 667-676, 2004

21. Corvinus FM, Orth C, Moriggl R, et al: Persistent STAT3 activation in colon growth. Neoplasia 7: 545-555, 2005.

22. Sherry MM, Reeves A, Wu JK and Cochran BH: STAT3 is required for proliferation and maintenance of multipotency in glioblastoma stem cells. Stem Cells 27: 2383-2392, 2009.

23. Bassères DS and Baldwin AS: Nuclear factor-kappaB and inhibitor of kappaB kinase pathways in oncogenic initiation and progression. Oncogene 25: 6817-6830, 2006.

24. Lam LT, Davis RE, Pierce J, et al: Small molecule inhibitors of IkappaB kinase are selectively toxic for subgroups of diffuse large B-cell lymphoma defined by gene expression profiling. Clin Cancer Res 11: 28-40, 2005.

25. Weis SM and Cheresh DA: Tumor angiogenesis: molecular pathways and therapeutic targets. Nat Med 17: 1359-1370, 2011.

26. Byun HJ, Lee JH, Kim BR, et al: Anti-angiogenic effects of thioridazine involving the FAK-mTOR pathway. Microvasc Res 84: 227-234, 2012.

27. Lechertier T and Hodivala-Dilke K: Focal adhesion kinase and tumour angiogenesis. J Pathol 226: 404-412, 2012.

28. Ponti D, Costa A, Zaffaroni N, et al: Isolation and in vitro propagation of tumorigenic breast cancer cells with stem/progenitor cell properties. Cancer Res 65: 5506-5511, 2005. 